\title{
Efficient Reversible Multiplexer Design Using proposed All- Optical New Gate
}

\author{
Amoldeep Singh ${ }^{1}$, Divya Dhawan ${ }^{2}$ \\ ${ }^{I}$ (PG Scholar, Department of electronics and communication engineering, Pec University of technology \\ Chandigarh, India) \\ ${ }^{2}$ (Assistant Professor, Department of electronics and communication engineering, Pec University of technology \\ Chandigarh, India)
}

\begin{abstract}
The interest for transmission capacity in overall information systems keeps on expanding because of developing utilization of Internet and high-transfer speed applications, for example, video. All-optical signal processing is one promising system for giving the essential limit and offers payload transparency, power utilization which scales proficiently with expanding bit rates, lessened handling inertness, and ultrafast signal processing. These networks do not have any optical to electrical converters because signal is photonic throughout its path and thus energy is conserved which is otherwise wasted in conversion of photons to electrons and back. Thus to achieve this, various configurations of Mach-Zehnder Interferometers are used which make signal processing quite fast and also power consumption is reduced. For all-optical switching to become a reality, integration is necessary to significantly reduce the cost of manufacturing, installation, and operation. One promising integrated all-optical logic gate is the semiconductor optical amplifier Mach-Zehnder interferometer (SOA-MZI) which has advantages like faster switching time, ease of fabrication, lesser power and high speed. As transistor count is increasing day by day, power consumption is increased so it has put a limit on transistor count. Therefore reversible logic has come into picture which has remarkable property of dissipating lesser power. So digital circuits are designed with only consists of reversible logic gates and thus researchers are aiming at designing new gates to reduce the optical cost and delay of the circuits. In this paper a new gate has been proposed and multiplexer design is shown with improved optical cost and delay and also various standard Boolean functions are also implemented using this new gate.
\end{abstract}

Keywords: All-Optical Communication, Mach-Zehnder Interferometer, Multiplexer, Reversible gates, Semiconductor optical amplifiers.

\section{Introduction}

With the progressions on the All-optical processing innovation, researchers have been giving careful consideration towards outlining low-power applications. All-optical computing implies there will be no opticalelectrical-optical converters required in the outline and signal will remain photonics all through its way. Favourable position of this will be that, the expanding transfer speed necessities will be met; furthermore $30 \%$ of energy will be spared which is generally wasted to change over electrons to protons and afterward back to electrons. Low power designs are likewise required in light of the fact that as indicated by Gordon Moore [1], quantities of transistors on an Integrated circuit (IC) are multiplying at regular intervals. Reversible logic plays an important role in reducing or even eliminating the power dissipation in a circuit because there is no loss of information in processing the data and original data can be retrieved at any stage of computation. These are special type of logic functions where input vectors are one-to-one mapped with the output vectors i.e. the number of inputs will be same as the number of outputs [2]. The concept of reversibility was first introduced by R.LAUNDAEUR [3] and C.H BENNETT [4]. According to laundaeur, every irreversible bit will dissipate energy in order of kT which later came out to be kTln2 joules. So researchers are focussing on designing logic gates which are reversible and also all-optical in nature.

Various reversible logic gates like Feynman gate, Toffoli gate, Peres gate, Fredkin gate etc, already exist in literatures which are designed using SOA-MZI switch [5-8]. These gates have been designed composed considering the parameters such as MZI switch count in designed circuit, number of beam splitters (BS), beam combiners (BC) and Optical delay which are also termed as cost parameters. Optical cost and delay for BC and BS are negligible, so it is not taken into account while calculating the overall optical cost and delay of the circuit. Several combinational circuits have been designed using these gates and are analysed in terms of cost parameters stated above. Attempt has been made by several researchers to reduce the MZI switch count and delay of these existing circuits by designing new gates or modifying the existing gates. One such attempt is made in this paper by designing a new all-optical reversible gate and improved designs of multiplexer is shown and verified using VPI Photonics tool. 


\section{Reversible Logic}

Reversibility implies that there is bijective mapping between the information and output vectors furthermore number of inputs ought to be equivalent to number of outputs i.e. $\mathrm{I}_{v}=\mathrm{O}_{\mathrm{v}}$. To keep up reversibility constant inputs and garbage outputs are required [6-8]. The consistent contribution to the reversible circuit is likewise called as ancilla inputs. Both ancilla inputs and garbage outputs are a vital piece of reversible circuit. They are essential to understand any logic function. Accordingly minimizing the reversible gate count and garbage output produced are prime objectives in any reversible circuit. There are two restrictions that should be dealt with while understanding a reversible circuit. Firstly fan out is not permitted, i.e. outputs from one phase can't be utilized as contribution to the following stage. Furthermore loops are not permitted in combinational circuits.

\section{All-Optical MZI Switch Architecture}

Mach-zehnder Interferometer is a device which calculates the phase shift introduced between the arms of the interferometer with the help of change in refractive index of the medium. When two SOA's are introduced in two arms of the interferometer as shown in figure 1, this architecture becomes SOA-MZI switch architecture which has many advantages like high speed, low power compact size and ease of fabrication because of the property of non-linearity of SOA.

This structure has two input ports and two output ports. First input port is given incoming signal and the second input port is given control signal both having different wavelengths and different laser power levels. It also has input coupler (50:50) to combine both the signals and split them into two arms of the interferometer and an output coupler (50:50) is used to later recombine these signals and split them into output channels. Semiconductor optical amplifiers use a semiconductor for providing the gain medium. They amplify the input signal without first converting them into electrical signals i.e. directly the optical signal is amplified. Switching of incoming signal depends on the saturation of two semiconductor optical amplifiers (SOA's) which further depends on the amount of light entering into arms.

The principle on which this switch works is explained as follows:

- When both input ports have light i.e. when control signal is present, it saturates the SOA-1 and changes its refractive index which is given by $\Delta \mathrm{n}=\mathrm{n}$.I where $\mathrm{n}$ is the refractive coefficient and I the intensity of light incident [5]. SOA-2 is still unsaturated thus the light will be present on the upper channel i.e. bar port and no light will be present at the lower channel.

- When incoming signal is high and control signal is low, both SOA's will be unsaturated thus no light will be present at the upper channel. Only the lower channel i.e. cross port receives light

- In all other cases there will be no light at any of the output channels.

- To block the control signal blocking filters (F) are placed at both the output ports.

The logic value 0 and 1 represent the absence and presence of light respectively. This behaviour of MZI switch can be represented as

- $\quad \mathrm{X}($ upper channel $)=P \cdot Q$

- $\quad \mathrm{Y}($ lower channel $)=\mathrm{P} . \overline{\mathrm{Q}}$

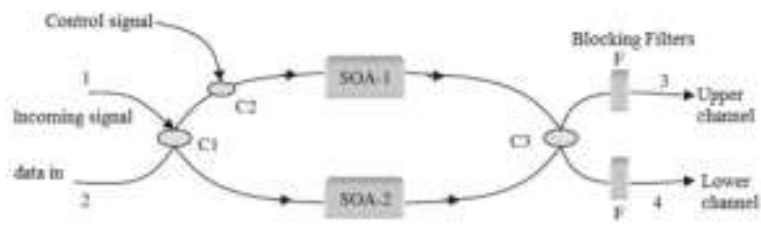

Fig.1 SOA-MZI switch architecture [5]

\section{Existing Gates in Literature}

A circuit is said to be reversible if it is built of cascade of reversible logic gates and there cannot be any feedbacks or fan outs permitted. Basic building blocks are reversible logic gates, which are built in optical domain using SOA-MZI switch. There are many basics gates which already exist in literature like Feynman gate, Toffoli gate, Peres gate etc which can produce any Boolean function and are discussed below.

\section{All Optical Feynman Gate}

Feynman gate is the most basic reversible gate having simple XOR operation with two inputs and two outputs. If Input vectors are $\mathrm{I}=(\mathrm{P}, \mathrm{Q})$ then corresponding output vector will be $\mathrm{O}=(\mathrm{X}=\mathrm{P}, \mathrm{Y}=\mathrm{P} \oplus \mathrm{Q})$. This gate can also work as a buffer if input $\mathrm{Q}=0$ and as a NOT gate if input $\mathrm{Q}=1$. Design of $2 \times 2$ Feynman gate using MZI switch is shown below in figure 2 . 


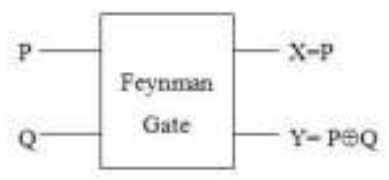

(a)

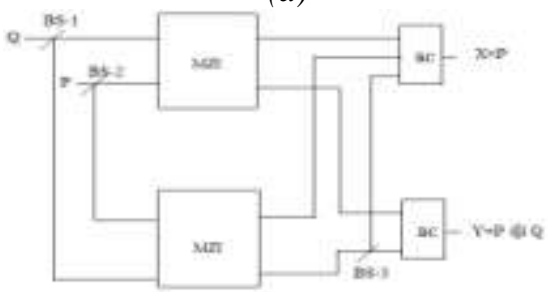

(b)

Fig.2 (a) Feynman Gate (b) Schematic using SOA-MZI switch [7]

In this gate MZI switch count came out to be 2, therefore it has optical cost of 2. Number of beam combiners (BC) used are 2 and number of beam splitters (BS) used are 3 and optical delay of this gate came out to be $1 \Delta$ as both MZI switches works in parallel.

\section{All Optical Toffoli Gate}

Toffoli gate is a $3 \mathrm{X} 3$ reversible gate with basic operation again as $\mathrm{XOR}$. If the input vector is $\mathrm{I}=(\mathrm{P}, \mathrm{Q}$, $R)$ then the corresponding output vector for this gate will be $\mathrm{O}=(\mathrm{X}=\mathrm{P}, \mathrm{Y}=\mathrm{Q}, \mathrm{Z}=\mathrm{P} . \mathrm{Q} \oplus \mathrm{R})$. Design of $3 \mathrm{X} 3$ Toffoli gate using MZI switch is shown below in figure 3.

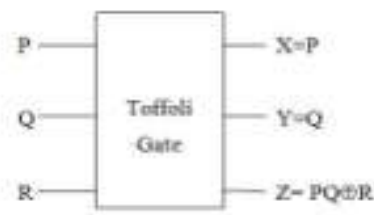

(a)

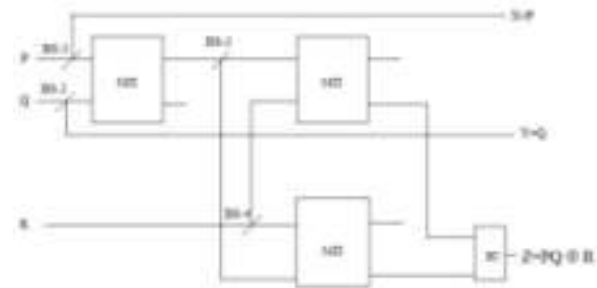

(b)

Fig.3 (a) Toffoli gate (b) Schematic using SOA-MZI switch [7]

So it is observed that MZI switch count came out to be 3, therefore its optical cost is 3 . Number of beam combiner (BC) used is 1 and number of beam splitters (BS) used are 4. Optical delay of this gate is $2 \Delta$ as the last two MZI switches work in parallel. This gate can also work as nand gate as shown in figure 3(b). If the input $\mathrm{C}$ is given logic 1 value thus output comes out to be NOT (AB).

\section{All Optical Peres Gate}

Peres gate is also a $3 \times 3$ reversible gate whose input vector is $\mathrm{I}=(\mathrm{P}, \mathrm{Q}, \mathrm{R})$ and the corresponding output vector will be $\mathrm{O}=(\mathrm{X}=\mathrm{P}, \mathrm{Y}=\mathrm{P} \oplus \mathrm{Q}, \mathrm{Z}=\mathrm{PQ} \oplus \mathrm{R})$. Design of $3 \times 3$ Peres gate using MZI switch is shown below in figure 4 .

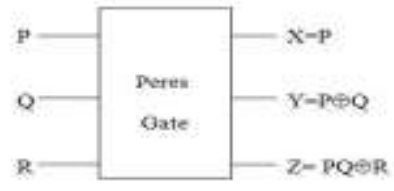

(a) 


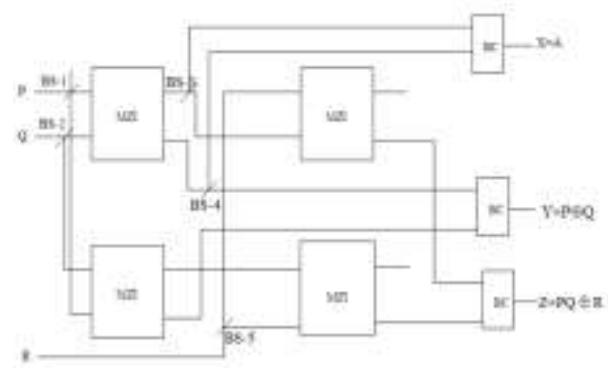

(b)

Fig.4 (a) Peres Gate (b) Schematic using SOA-MZI switch [7]

In this gate MZI switch count comes out to be 4, therefore its optical cost is 4 . Number of beam combiners (BC) used are 3 and number of beam splitters (BS) used are 5. Optical delay of this gate is $2 \Delta$ as the last two pairs of MZI switches work in parallel.

\section{Proposed All-Optical New Gate}

This section briefly describes the structure of new gate using SOA-MZI switch. Figure 5 has shown $3 \times 3$ new all-optical reversible gate. If input vector is $\mathrm{I}=(\mathrm{P}, \mathrm{Q}, \mathrm{R})$ then the corresponding output vector will be $\mathrm{O}=(\mathrm{X}=\mathrm{PQ}+\overline{\mathrm{QR}}, \mathrm{Y}=\mathrm{P}, \mathrm{Z}=\mathrm{P} \oplus \mathrm{Q} \oplus \mathrm{R})$

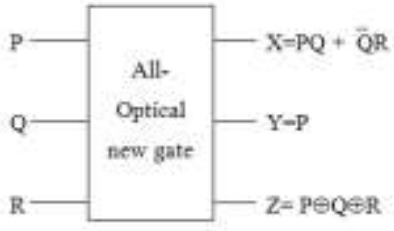

(a)

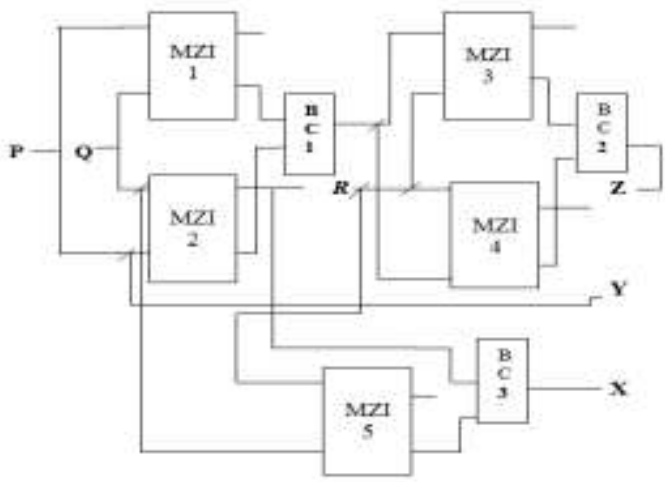

(b)

Fig.5 (a) All-Optical new gate (b) Schematic using SOA-MZI switch

This gate consists of $5 \mathrm{MZI}$ switches which are as follows:

- $\quad$ Four MZI switches to implement $\mathrm{P} \oplus \mathrm{Q} \oplus \mathbb{R}$ thus optical cost for this implementation will be 4.

- $\quad$ One MZI switch to implement $\mathrm{X}=\mathrm{RQ}+\overline{\mathrm{QP}}$.

Therefore total optical cost of the circuit is 5 and optical delay is $2 \Delta$ as three pairs of MZI switch works in parallel.

\section{Propose Multiplexer Design Using New Gate}

This gate can also be used as $2 \times 1$ multiplexer considering inputs $\mathrm{P}$ and $\mathrm{R}$ as data inputs and $\mathrm{Q}$ as a select line. Thus the output $\mathrm{X}$ will be the output of this multiplexer.

- When $\mathrm{Q}=0, \mathrm{R}$ will be selected and produced at the output.

- When $\mathrm{Q}=1, \mathrm{P}$ will be selected and produced at the output.

- Since new gate has MZI switch count as 5, thus optical cost of $2 x 1$ multiplexer is 5 and optical delay is $3 \Delta$ because the two pairs of MZI switch work in parallel. 


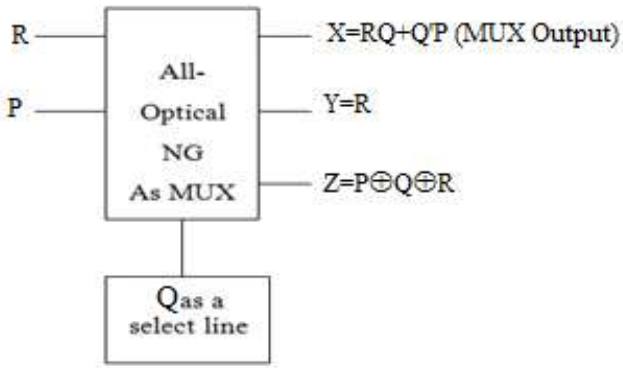

Fig.6 $2 \times 1$ multiplexer using new gate

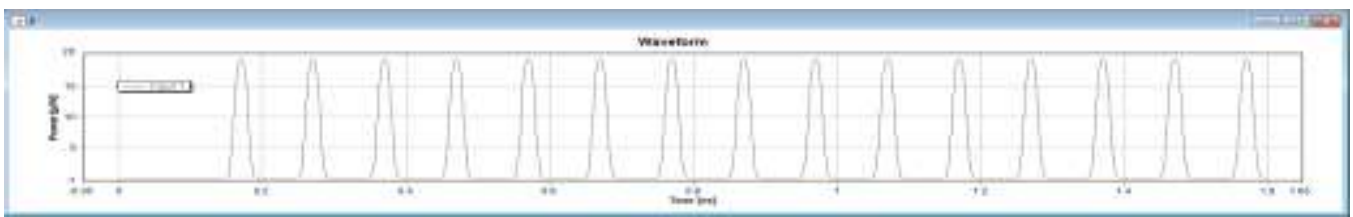

Fig.7 (a) Input $\mathrm{P}$

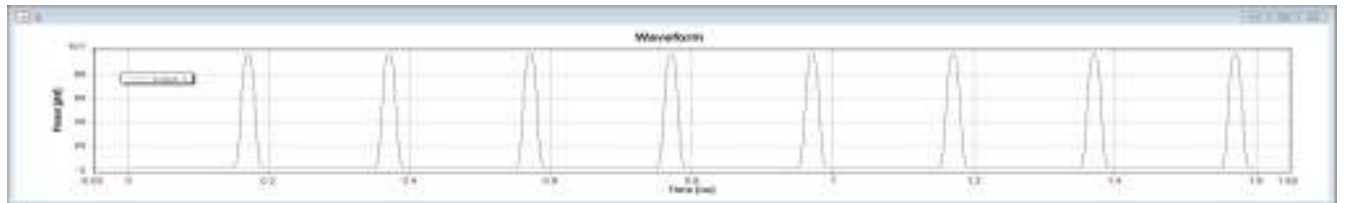

Fig.7 (b) Input Q as a select line

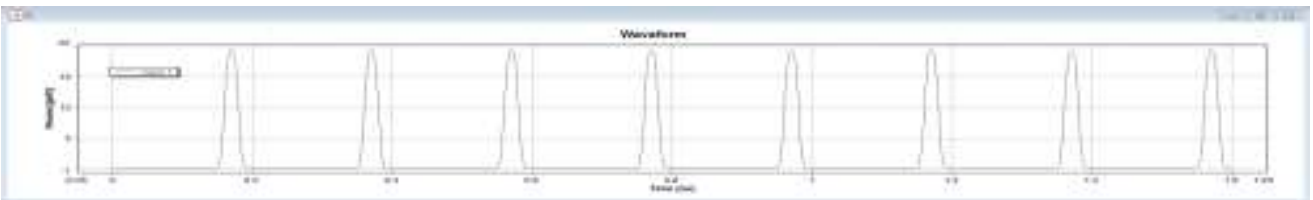

Fig.7 (c) Input R

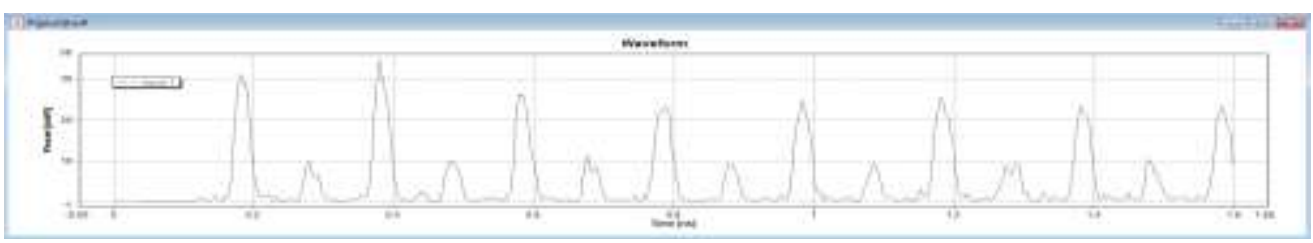

Fig.7 (d) 2x1 MUX output

\section{Results and Discussions}

$2 \times 1$ Multiplexer is designed using proposed new All-optical reversible gate with improved cost. The above waveforms shown in Fig.7 (a), (b), (c), (d) verify the functionality of these circuits. Talking about the previous multiplexer design which were implemented in [11] and [12], our design is improved in terms of optical cost and delay and a comparison has been done in Table 1. Also beam splitters and beam combiners are taken into account in the following comparison.

Table 1: Comparative Study of All-Optical Reversible 2x1 Multiplexer

\begin{tabular}{|l|l|l|l|l|}
\hline Proposed Design & MZI Switch Count & Optical Delay & BS & BC \\
\hline Our Design & 5 & $3 \Delta$ & 5 & 3 \\
\hline \multirow{2}{*}{ G.K Maity et al. [12] } & 8 & $3 \Delta$ & 12 & 5 \\
\cline { 2 - 5 } & $\{\mathbf{3 7 . 5 \%}\}$ & No IP & $\{\mathbf{5 8 . 3 3 \%}\}$ & $\{\mathbf{4 0 \%}\}$ \\
\hline \multirow{2}{*}{ M.B Malav et al.[11] } & 7 & $3 \Delta$ & 8 & 3 \\
\cline { 2 - 5 } & $\{\mathbf{2 8 . 5 7 \%}\}$ & No IP & $\{\mathbf{3 7 . 5 \%}\}$ & No IP \\
\hline
\end{tabular}

From the above table significant improvements have been seen in terms of optical cost, number of beam combiners and number of beam splitters. The proposed new reversible gate can also be used to implement reversible Boolean functions. If we talk about three variables A, B, C then the possible combinations will be $2^{3}$. 
For these combinations there are $2^{8}$ Boolean functions possible. Thus 13 standard Boolean functions are implemented with this new gate. Compared to previous implementation using NOR based gates and All-optical Toffoli gate, analysis has been done in terms of optical cost and delay in table 2 . The proposed gate has reduced the cost as compared to existing Toffoli gate and TNOR gate.

Table 2: Optical Cost and Delay Analysis of Reversible New Gate by Implementing 13 Standard Boolean

\begin{tabular}{|c|c|c|c|c|c|c|c|}
\hline \multicolumn{8}{|c|}{ Functions } \\
\hline \multirow[t]{2}{*}{$\begin{array}{l}\text { Function } \\
\text { no. }\end{array}$} & \multirow[t]{2}{*}{ Standard Functions } & \multicolumn{2}{|c|}{$\begin{array}{l}\text { Implementation } \\
\text { using All-Optical } \\
\text { Toffoli gate }\end{array}$} & \multicolumn{2}{|c|}{$\begin{array}{l}\text { Implementation } \\
\text { using All-Optica } \\
\text { TNOR Gate }\end{array}$} & \multicolumn{2}{|c|}{$\begin{array}{l}\text { Implementation using } \\
\text { Proposed New gate }\end{array}$} \\
\hline & & $\begin{array}{l}\text { Optical } \\
\text { Cost }\end{array}$ & $\begin{array}{l}\text { Optical } \\
\text { Delay }\end{array}$ & $\begin{array}{l}\text { Optical } \\
\text { Cost }\end{array}$ & $\begin{array}{l}\text { Optical } \\
\text { Delay }\end{array}$ & $\begin{array}{l}\text { Optical } \\
\text { Cost }\end{array}$ & $\begin{array}{l}\text { Optical } \\
\text { Delay }\end{array}$ \\
\hline 1 & $\mathrm{~F}=\mathrm{ABC}$ & 6 & $4 \Delta$ & 10 & $3 \Delta$ & 10 & $4 \Delta$ \\
\hline 2 & $\mathrm{~F}=\mathrm{AB}$ & 3 & $2 \Delta$ & 6 & $2 \Delta$ & 5 & $2 \Delta$ \\
\hline 3 & $\mathrm{~F}=\mathrm{ABC}+\mathrm{AB}^{\prime} \mathrm{C}^{\prime}$ & 19 & $10 \Delta$ & 16 & $5 \Delta$ & 10 & $4 \Delta$ \\
\hline 4 & $\mathrm{~F}=\mathrm{ABC}+\mathrm{A}^{\prime} \mathrm{B}^{\prime} \mathrm{C}^{\prime}$ & 21 & $9 \Delta$ & 16 & $5 \Delta$ & 20 & $4 \Delta$ \\
\hline 5 & $\mathrm{~F}=\mathrm{AB}+\mathrm{BC}$ & 9 & $6 \Delta$ & 6 & $2 \Delta$ & 10 & $4 \Delta$ \\
\hline 6 & $\mathrm{~F}=\mathrm{AB}+\mathrm{A}^{\prime} \mathrm{B}^{\prime} \mathrm{C}$ & 16 & $9 \Delta$ & 14 & $4 \Delta$ & 15 & $4 \Delta$ \\
\hline 7 & $\mathrm{~F}=\mathrm{ABC}+\mathrm{A}^{\prime} \mathrm{BC}^{\prime}+\mathrm{AB}^{\prime} \mathrm{C}^{\prime}$ & 30 & $15 \Delta$ & 20 & $6 \Delta$ & 10 & $4 \Delta$ \\
\hline 8 & $\mathrm{~F}=\mathrm{A}$ & 3 & $2 \Delta$ & 2 & $1 \Delta$ & 5 & $2 \Delta$ \\
\hline 9 & $\mathrm{~F}=\mathrm{AB}+\mathrm{BC}+\mathrm{AC}$ & 15 & $10 \Delta$ & 14 & $4 \Delta$ & 10 & $4 \Delta$ \\
\hline 10 & $\mathrm{~F}=\mathrm{AB}+\mathrm{B}^{\prime} \mathrm{C}$ & 11 & $5 \Delta$ & 12 & $3 \Delta$ & 5 & $2 \Delta$ \\
\hline 11 & $\mathrm{~F}=\mathrm{AB}+\mathrm{BC}+\mathrm{A}^{\prime} \mathrm{B}^{\prime} \mathrm{C}^{\prime}$ & 24 & $9 \Delta$ & 12 & $4 \Delta$ & 10 & $4 \Delta$ \\
\hline 12 & $\mathrm{~F}=\mathrm{AB}+\mathrm{A}^{\prime} \mathrm{B}^{\prime}$ & 13 & $5 \Delta$ & 10 & $3 \Delta$ & 5 & $2 \Delta$ \\
\hline 13 & $\mathrm{~F}=\mathrm{ABC}+\mathrm{A}^{\prime} \mathrm{B}^{\prime} \mathrm{C}+\mathrm{AB}^{\prime} \mathrm{C}^{\prime}+\mathrm{A}^{\prime} \mathrm{B} \mathrm{C}^{\prime}$ & 39 & $11 \Delta$ & 28 & $6 \Delta$ & 5 & $2 \Delta$ \\
\hline \multicolumn{2}{|l|}{ TOTAL } & 209 & $97 \Delta$ & 166 & $48 \Delta$ & 120 & $40 \Delta$ \\
\hline \multicolumn{4}{|c|}{ \% Improvement } & $20.57 \%$ & $50.52 \%$ & $27.71 \%$ & $16.66 \%$ \\
\hline
\end{tabular}

\section{Conclusion}

In this work, a new All-Optical reversible gate has been proposed. Using this gate an efficient multiplexer design has been proposed and its functionality is verified in VPI Photonics software. This gate has reduced the cost of multiplexer design significantly as compared to existing designs and improvement is shown in tabular form. Also the 13 standard Boolean functions are implemented using this gate and compared with toffoli gate and NOR gate implementations. Comparison results are shown in tabular form and we came out with significant improvements in terms of optical cost and delay. Furthermore many digital circuits can be designed with this gate with improved designs and cost. Future work will concentrate on designing new gates which are much efficient that previously designed gates.

\section{References}

[1]. [1] Moore G, "The Future of Integrated Electronics", Proceedings of IEEE, 1965, January; 38(8).

[2]. [2] Toffoli T, "Reversible computing", Tech. Memo-MIT/LCS/TM-151, MIT Lab for Comp. Sci, 1980.

[3]. [3] Landauer R, "Irreversibility and heat generation in the computing process", IBM Journal of Research and Development, 1961, July; 4(3):183-191

[4]. [4] C. H. Bennett "Logical reversibility of computation", IBM Journal of Logical Research and Development, 1973, November; 17(6):525-532

[5]. [5] Taraphdar Chinmoy, Chattopadhyay Tanay, Roy Jitendra Nath, "Mach-zehnder interferometer-based all-optical reversible logic gate", Optics and Laser Technology, 2010; 42(2): 249-259.

[6]. [6] Fredkin E and Toffoli T, “Conservative logic”, International J.Theor.Physics, 1982; 21:219-253.

[7]. [7] Kotiyal S, Thapliyal H and Ranganathan N, "Mach-Zehnder Interferometer based All-optical reversible NOR Gates", IEEE Computer Society Annual Symposium on VLSI, 2012, Aug, 19-21:207-212.

[8]. [8] Maity G. K, Roy J. N, and Maity S. P, "Mach-zehnder interferometer based all-optical peres gate", Advances in Computing and Communications, 2011; 192:249-258.

[9]. [9] Saeedi M and Markov Igor L, "Synthesis and Optimization of Reversible Circuits - A Survey", ArXiv: 1110.2574v2 [cs.ET], 2013, March, 20:1-34.

[10]. [10] Zhang M, Zhao Y, Wang L, Wang J, Ye P, "Design and analysis of all-optical XOR gate using SOA-based Mach-Zehnder Interferometer", Optics Communications, 2003; 223:301-308.

[11]. [11] Malav M.B, Gupta S and Jain S.C, "A New Gate for Low Cost Design of All-optical Reversible Logic Circuit", International Conference on Circuit, Power and Computing Technologies [ICCPCT], 2015.

[12]. [12] Maity G.K, Chattopadhyay T, Roy J.N and Maity S.P, "All-optical reversible multiplexer", International conference on Computers and Devices for Communication (CODEC), 2009.

[13]. [13] Maity G. K, Maity S. P, Chattopadhyay T and Roy J. N, "Mach-Zehnder Interferometer Based All-Optical Fredkin Gate", International Conference on Trends in Optics and Photonics, Kolkata, 2009, March, 1 - 4.

[14]. [14] Maity G. K, Roy J. N and Maity S.P, "Design of all-optical new gate using Mach-Zehnder interferometer", International Conference on Devices, Circuits and Systems (ICDCS), 2012, March, 15-16:474-478.

[15]. [15] Maity G. K and Maity S. P, "Implementation of HNG using MZI", Third International Conference on Computing Communication \& Networking Technologies (ICCCNT), 2012, July, 26-28:1-6. . 
[16]. [16] Chattopadhyay T, “All-optical modified Fredkin gate”, Selected Topics in Quantum Electronics, IEEE Journal, 2011; 18(99):1 -8 .

[17]. [17] Mandal A.K and Maity G.K, "An All-optical New Universal Gate Using Mach-Zehnder Interferometer, IEEE, Sixth International Conference on Computational Intelligence and Communication Networks, 2014:1042-1046.

[18]. [18] Datta Kamalika, Sengupta Indranil, "All-Optical Reversible Multiplexer design using Mach-Zehnder Interferometer, IEEE, international conference on Computer Society Digital Library (CSDL), 2014:539-544. 